\title{
Une révolution médicale? Dynamiques des professions de santé entre révolution et empire
}

A medical Revolution? The dynamics of the medical professions between

Revolution and Empire

\section{Christelle Rabier}

\section{(2) OpenEdition}

\section{Journals}

\section{Édition électronique}

URL : https://journals.openedition.org/ahrf/11490

DOI : 10.4000/ahrf.11490

ISSN : 1952-403X

Éditeur :

Armand Colin, Société des études robespierristes

Édition imprimée

Date de publication : 1 janvier 2010

Pagination : 141-159

ISSN : 0003-4436

Référence électronique

Christelle Rabier, « Une révolution médicale ? Dynamiques des professions de santé entre révolution et empire », Annales historiques de la Révolution française [En ligne], 359 | janvier-mars 2010, mis en ligne le 01 mars 2013, consulté le 22 avril 2022. URL : http://journals.openedition.org/ahrf/11490 ; DOI : https://doi.org/10.4000/ahrf.11490 


\title{
UNE RÉVOLUTION MÉDICALE? DYNAMIQUES DES PROFESSIONS DE SANTÉ ENTRE RÉVOLUTION ET EMPIRE
}

Christelle RABIER

\begin{abstract}
Au terme d'une décennie de réglementations sur les modes de qualification des professionnels de santé, la loi de ventôse an XI fonde un nouvel ordre médical qui stipule l'unité de l'art de guérir et propose une organisation professionnelle déterminée par le diplôme. Cet ordre idéal est mis en œuvre par l'administration impériale, qui cherche à unifier sous son autorité les multiples sources de légitimité médicale pendant l'Ancien Régime. Je me propose de rendre compte de la construction d'une nouvelle profession médicale qui intègre en son sein des praticiens formés et qualifiés de 1746 à 1806 . En premier lieu, la liste dévoile l'organisation du travail au XVIII siècle, partiellement transformée après l'an XI. Vilipendée, la classe formée entre 1792 et l'an XI, présente des caractéristiques hors normes : non diplômés, les praticiens autorisés par l'administration sont certifiés par des notables - légitimation recevable au regard des modes des normes professionnelles du temps.
\end{abstract}

Mots-clés : profession, officier de santé, docteur en médecine, docteur en chirurgie, sage-femme, certification professionnelle.

Le $1^{\mathrm{er}}$ avril 1807, Nicolas Frochot (1761-1828), premier préfet du département de la Seine, arrête la « Liste des médecins, chirurgiens, docteurs en médecine et en chirurgie, officiers de santé et sages-femmes qui ont fait enregistrer leurs titres aux secrétariats de la préfecture et des deux sous-préfectures du département de la Seine $»^{1}$. Il s'agit d'un tableau com-

(1) Bibliothèque nationale de France (dorénavant BNF), 4-T9-35, Préfecture de la Seine, Liste des Médecins, Chirurgiens, Docteurs en médecine et en chirurgie, Officiers de santé et SagesFemmes qui ont fait enregistrer leurs titres aux Secrétariats de la Préfecture et des deux sous- 
posite, par lequel l'administration impériale cherche à unifier sous son autorité les multiples sources de légitimité médicale. Sa finalité est bien de les conserver toutes, de n'en exclure aucune, mais de les unifier et par là de leur restituer une légitimité nouvelle. Plus de quinze ans après la suppression des jurandes (1791) et des sociétés savantes (1793), l'abrogation des métiers de médecin et de chirurgien est exemplaire des transformations promues par le pouvoir impérial : d'une part, qualifiés « [d']anciens », ils sont relégués dans un passé révolu; d'autre part, le nom même du métier a disparu des nouvelles formes qui séparent les docteurs en médecine et en chirurgie des officiers de santé.

Alors que la loi de ventôse an XI entend clarifier une situation professionnelle complexe, l'état des praticiens que dresse l'administration préfectorale met en évidence la multiplicité des statuts qui régissent les professions de santé au début du XIX ${ }^{\mathrm{e}}$ siècle. En les unifiant, l'administration de l'Empire modifie la hiérarchie des métiers en mettant en place un système où les professions sont subordonnées au diplôme. Elle transforme également, de façon durable, les formes de certification professionnelle, en redoublant auprès de l'administration la qualification qui était jusque là uniquement assurée localement par les pairs, dans les Facultés de médecine ou les Collèges de chirurgie. Ce faisant, en précisant l'origine de l'accession aux métiers des praticiens, le document donne à lire le résultat d'un siècle de construction professionnelle et de son organisation dans la ville : l'inscription des praticiens anciens et nouveaux est l'occasion de faire apparaître de nombreux salariés de la santé qui étaient invisibles dans les sources des corporations.

La liste préfectorale de 1807 permet de poser à nouveaux frais la question des mutations qui se sont opérées chez les praticiens de santé au cours des années révolutionnaires. Elle ignore clairement de nombreux métiers de la santé, comme les garde-malades ou les infirmiers, mais elle donne accès à des groupes professionnels exerçant la médecine et la chirurgie quelquefois ignorés des historiens de la médecine. En étudiant le cas particulier des médecins et des chirurgiens, je me propose de rendre compte de la construction d'une nouvelle profession médicale qui intègre en son sein des praticiens formés et qualifiés de 1746 à 1806. En premier lieu, j'étudierai l'organisation sociale de l'Ancien Régime et met-

préfectures du Département de la Seine, Paris, Impr. de Ballard, 1807; URL : http://web2.bium.univparis5.fr/livanc/?cote $=08233 \times 09 \&=1 \&$ do $=$ pagei, numérisé par la Bibliothèque Interuniversitaire de Médecine (dorénavant Bium), consultation le 20 octobre 2008. L'exemplaire de la BNF comporte un supplément à la liste publiée le $1^{\text {er }}$ avril, daté du 20 juin 1807, dont j'ai tenu compte. 
trai en évidence comment l'administration impériale met au grand jour le marché du travail médical au XVIII ${ }^{\mathrm{e}}$ siècle. Les projets et les décisions politiques qui se sont succédé pour refonder l'organisation de la santé en France aboutissent à l'élaboration de deux ordres de praticiens, les docteurs et les officiers de santé, catégories qui sont à leur tour investies par les débutants dans le métier. Vilipendée, la classe formée entre 1792 et l'an XI, présente des caractéristiques hors normes : non diplômés, les praticiens autorisés par l'administration sont certifiés par trois notables. Il s'agira de comprendre pourquoi ce mode de certification pouvait être recevable pour les acteurs, administratifs ou professionnels.

\section{L'organisation sociale de l’Ancien Régime médical}

Pour comprendre l'organisation du marché du travail médical sous l'Ancien Régime, seules subsistent les listes publiées par les corporations parisiennes, qui ne prennent en compte que les membres, maîtres ou docteurs-régents ${ }^{2}$. En sont exclus les praticiens nombreux, qui appartiennent bien à la profession - puisqu'ils sont par exemple chirurgiens reconnus comme tels dans le voisinage, comme en attestent les archives des commissaires par exemple - mais ils n'ont pas adhéré à la corporation. À la fin du XVIII ${ }^{\mathrm{e}}$ siècle, la Société royale de médecine, puis le Comité de salubrité, qui ont cherché à enquêter sur les provinces pour en connaître les « charlatans », n’ont pas étudié la capitale parisienne ${ }^{3}$.

(2) Pour les chirurgiens, AN, Y 11860, «Compte de la recette et dépense faittes pour le Collège Royal de St. Côme à Paris par M. Pierre Rival, maître en chirurgie, ancien prévôt, receveur et comptable », $1^{\text {er }}$ octobre 1757-1 ${ }^{\text {er }}$ novembre 1758; BNF T9-1, Liste de Messieurs les Chirurgiens Jurez de la Paris, 1730-1783 (s.n.) : la BNF conserve une liste annuelle des «maîtres en l'art et science de chirurgie et de l'Académie royale » de 1760 à 1783, à l'exception des années 1762-1763, 1766-1767, 1770-1772, et 1780. Pour les médecins, voir Isabelle CoQUiLlard, Les docteurs-régents de la Faculté de médecine de Paris au XVII et XVIII siècles, Thèse de doctorat, Université de Paris-X Nanterre (en cours).

(3) Jean-Pierre Goubert, « The Extent of Medical Practice in France around 1780 », Journal of Social History, 10, 1976-1977, p. 410-427; Jean-Pierre GouBERT et Bernard LePETIT, « Les niveaux de médicalisation des villes françaises à la fin de l'Ancien Régime », dans Jean-Pierre GouBERT, $L a$ médicalisation de la société française, Waterloo, Ont., Historical Reflections Press, 1982, p. 45-67. Pour la France, Goubert s'est fondé sur les deux enquêtes de la fin du XVIII ${ }^{\mathrm{e}}$ siècle, celle du Contrôleur général Calonne, assisté de la Société royale de médecine de 1786, menant l'enquête sur six généralités - Amiens, Caen, Dijon, Rennes, Soissons et Tours - et sur celle du Comité de salubrité de l'Assemblée constituante, qui s'appuie alors sur le réseau des lieutenants du premier chirurgien du roi : AN, F15225 à 2282; AN, F17 2276; Toby Gelfand, « Medical Professionals and Charlatans. The Comité de Salubrité Enquête of 1790-91 », Histoire sociale - Social History, 11, 1978, p. 62-97. Singulièrement, elles ont été étudiées sous l'angle du charlatanisme, entendu objectivement comme exercice illégal ou dangereux de la médecine et non pas de façon critique, comme lieu de fixation de normes professionnelles et administratives. 
Aussi la « Liste des médecins, chirurgiens, docteurs en médecine et en chirurgie, officiers de santé et sages-femmes qui ont fait enregistrer leurs titres » publiée par la Préfecture de police en 1807 constitue-t-elle un document particulièrement précieux. Elle résulte d'une disposition de la loi du 19 ventôse an XI, qui stipule que les praticiens en exercice sous l'Ancien Régime doivent se faire enregistrer auprès de leurs institutions locales :

« Les médecins ou chirurgiens, établis depuis la suppression des universités, facultés, collèges et communautés, sans avoir pu se faire recevoir, et qui exercent depuis trois ans, se muniront d'un certificat délivré par les sous-préfets de leurs arrondissements, sur l'attestation du maire et de deux notables des communes où ils résident, au choix des sous-préfets : ce certificat, qui constatera qu'ils pratiquent leur art depuis l'époque indiquée, leur tiendra lieu de diplôme d'officier de santé $»^{4}$.

L'intérêt de cet acte administratif est double. D'une part, il recense tous les professionnels selon des critères explicites : métier, nom, prénom, adresse, complément d'adresse, mode de certification, date. Au total ce sont 743 praticiens et 165 sages-femmes qui se trouvent ainsi identifiés, voire localisés, par l'administration. D'autre part, il rend compte de l'intégralité des formes de qualification professionnelle ou de réception dans la profession entre 1746 et 1806. L'intérêt de la liste de 1807 est qu'elle reconnaît sur un même plan des hommes appartenant à l'élite des corps de métier et d'autres, au statut inférieur, et jusqu'alors invisibles dans les documents officiels. C'est ainsi une hiérarchie à multiples degrés qui organise les 164 médecins et 241 chirurgiens qualifiés sous l'Ancien Régime.

L'appartenance à une ancienne corporation parisienne ou provinciale - au nombre desquelles il faut compter les Facultés de médecine, les Collèges et les communautés de chirurgie - admet docteurs en médecine et maîtres en chirurgie dans la catégorie « officiers de santé ». La liste distingue les membres des prestigieux Facultés de médecine et Collège de chirurgie de Paris des autres praticiens, « anciens médecins » ou « anciens chirurgiens », qualifiés d'autre manière. Du seul Collège de chirurgie, qui compte après 1760 autour de 280 membres, subsistent 81 maîtres. La liste identifie les hommes inscrits «sur le calendrier du Collège en 1789 ».

(4) Loi du 19 ventôse an XI, article 23, cité par Jacques LÉONARD, La France médicale au XIX siècle, Paris, Gallimard-Julliard, 1978, p. 72. 
Un croisement avec l'Almanach royal pour l'année commune 1789-1790 indique que ces hommes ont bien fait partie du Collège; ils y étaient impétrants, l'accession à la maîtrise prenant plusieurs mois. La population a bénéficié d'une forte immigration : les médecins de province, venus de Montpellier, Reims et d'autres villes françaises, ont afflué au point de doubler le nombre de docteurs-régents de la Faculté de Paris en 1807. La mobilité des chirurgiens est moins nette : seuls dix maîtres venus pour l'essentiel du Bassin Parisien ont gagné la capitale sur les 81 subsistant de l'ancien Collège de Paris.

En séparant les « chirurgiens-dentistes » des « chirurgiens-herniaires », la Préfecture distingue les anciens « experts », titre que leur reconnaît le règlement de $1768^{5}$. S'il n'y a aucune ambiguïté sur le terme de dentiste, spécialité qui émerge au XVIII ${ }^{\mathrm{e}}$ siècle $^{6}$, les chirurgiens herniaires ou bandagistes sont moins connus : disposant d'une technologie propre, qui associe bandages et petite métallurgie pour traiter différentes affections comme les hernies, les descentes d'organes, voire les anus artificiels, ce métier apparu au cours du siècle précédent s'est vu agréger au Collège de chirurgie par arrêt royal ${ }^{7}$. Distinction de compétence, c'est également une distinction sociale que recouvre le titre d' « expert » : ces hommes, lors de l'agrégation, bénéficient de droits allégés et d'examens spécifiques $^{8}$. Bien qu'il n'y ait pas d'étanchéité des sous-groupes, ces spécialités disposent d'une certaine autonomie professionnelle?.

La préfecture accepte en outre les praticiens « reçus par charge »ce par quoi il faut comprendre le service pensionné des maisons royales ${ }^{10}$. Après l'octroi du règlement de 1768 , tous ces praticiens n'ont pas fait partie du Collège parisien, en dépit du rabais dont ils bénéficiaient sur les droits d'accession à la maîtrise et de la procédure simplifiée d'admission,

(5) Lettres patentes [...] portant règlement pour le Collège de chirurgie, Titre neuvième, « De la réception des experts », art. CXXVI-CXXIX. Il faut noter que la distinction terminologique qu'opère l'Empire indique que les métiers ont acquis une plus grande autonomie.

(6) Colin Jones, « Pulling Teeth in Eighteenth-Century Paris », Past and Present, 166, 2000, p. $100-145$.

(7) Statuts, reglemens, arrests du conseil d'État et lettres patentes portant confirmation de la Déclaration du roy du mois de novembre 1634, pour les maistres operateurs chirurgiens herniaires de la Ville et Fauxbourgs de Paris, Paris, s. n., 1696.

(8) Deux examens et près de 550 liv. - dont 300 livres pour la bourse commune - plus des jetons d'argent et des paires de gants : Ibid., art. CXLII.

(9) Ces deux spécialités ont pu être pratiquées par des maîtres en chirurgie : en effet, l'Almanach royal de 1789 indique que les chirurgiens Pipelet, demeurant rue Mazarine, sont également herniaires. Pipelet fils, reçu en 1786, loge encore à la même adresse en 1807.

(10) Alexandre Lunel, La Maison médicale du Roi, XVII ${ }^{e}$-XVIII ${ }^{e}$ siècles. Le pouvoir royal et les professions de santé (médecins, chirurgiens, apothicaires), Paris, Champ Vallon, 2008, p. 312-315. 
puisqu'ils sont soumis au seul examen public ou $«$ acte $»^{11}$. Au contraire, leur droit d'exercer dans la capitale, auprès d'une clientèle privée, sans compte à rendre au Collège de chirurgie, a continué de susciter un contentieux $^{12}$. Ce système parallèle de certification de praticiens médicaux persiste jusqu'à la République, comme en atteste le brevet de Laurent Bézaut, reçu chirurgien-major par le roi, le 27 mai 1792, ce qui lui donne lieu de titre ${ }^{13}$.

L'exercice par «baux de privilège » désigne la pratique d'un chirurgien auprès d'une veuve de maître à qui il a loué un droit d'exercer, placée sous la responsabilité de la corporation parisienne ${ }^{14}$. C'est la seule façon d'exercer légalement de façon indépendante, sans avoir été admis à exercer par le Collège ou par les maisons royales. Le nombre de privilégiés est fonction de la démographie des veuves : parmi les 18 chirurgiens qualifiés de la sorte entre 1777 et 1790, la moitié d'entre eux ont acquis leur bail de privilège après 1787. Cela laisse entrevoir la précarité statutaire dans laquelle se trouvent les chirurgiens privilégiés, qui est aussi une précarité judiciaire ${ }^{15}$.

Le sous-groupe des « chirurgiens reçus par le lieutenant du premier chirurgien du roi » mérite qu'on s'y attarde. Dès 1699, la réglementation oblige l'enregistrement des brevets d'apprentissage auprès des lieutenants du Premier chirurgien du roi ${ }^{16}$. Ce groupe, fort de 102 praticiens, soit

(11) Lettres-patentes du Roi en forme d'édit, portant règlement pour le Collège de chirurgie de Paris. Données à Versailles au mois de mai 1768. Registrées en Parlement le 10 mai 1768, Paris, Imprimerie royale, 1768, art. CXXII et CXXIV, soit 260 liv. Les maitres parisiens sont eux soumis à dix examens (immatricule, tentative, entrée en semaine, ostéologie, anatomie, opérations, médicaments, dernier examen et acte public), qui requièrent au total le paiement de 933 liv. auxquelles il faut ajouter 600 liv. de bourse commune payables en deux fois, la première avant l'immatricule, et divers dons de jetons d'argent et de paires de gants. Les fils de maîtres payent la moitié de ces droits : art. CXXXIX, CXL et CXLI.

(12) Le dernier en date remonterait à 1769 , après l'octroi du nouveau règlement de 1768 : BNF, T18-191 (39, 8, 121) : Requête présentée au Roy, par M. le Chirurgien ordinaire, \& MM. les huit Chirurgiens en Titre d'Office, servant par quartier auprès de Sa Majesté, Paris, Veuve d'Houry, 1769.

(13) On peut souligner qu'en l'absence de brevet, c'est l'Almanach royal qui fait foi, comme dans le cas d'Etienne Thibaut : cf. Liste des médecins..., op. cit., p. 8.

(14) La catégorie des veuves est encore très mal connue, qui plus est les veuves de chirurgiens : voir le travail de Janine M. LANza, From Wives to Widows in Early Modern Paris. Gender, Economy and Law, Aldershot, Ashgate, 2007.

(15) Sur l'affaire Callé, par lequel un chirurgien privilégié se trouve condamné à une lourde amende et interdiction d'exercer en appel auprès du Parlement de Paris, voir Christelle RABIER, Les chirurgiens de Paris et de Londres : économie, identités, savoirs, thèse de doctorat, Paris I, 2008, p. 83 et suiv.

(16) Statuts pour la communauté des maîtres chirurgiens jurez de Paris, Paris, veuve de Pierre et Louis Colin, 1701, art. XXXIX. 
près de $40 \%$ des anciens chirurgiens, dont leur doyen inscrit en 1746 , comprend donc des chirurgiens qui ont commencé leur éducation avant 1791 et n'ont pas eu le temps - ou n'ont pu faire le choix - soit d'accéder à la maîtrise, soit d'acquérir un bail de privilège. Il s'agit donc d'assistants ou de compagnons de maîtres, ou encore de praticiens « illégaux ». Leur origine largement parisienne s'explique par la très forte attraction de la capitale sur les impétrants de la chirurgie dans la seconde moitié $\mathrm{du} \mathrm{XVIII}^{\mathrm{e}}$ siècle, en raison des cours gratuits du Collège de chirurgie et des places ouvertes dans les hôpitaux parisiens ${ }^{17}$. Sans préjuger de leur éducation, les « chirurgiens reçus » ne diffèrent des anciens membres du Collège de Paris, non par l'expérience - la longévité du doyen Jollet est là pour le prouver - mais par le paiement de droits fort élevés.

Cette importante population de «garçons » légaux ou illégaux - dans le contentieux du Châtelet on trouve l'appellation plus connue de « chirurgiens chambrelans »- invite à reconsidérer le marché du travail sous l'Ancien Régime, ce qu'a manifestement occultée une historiographie qui voulait saisir l'intimité de la relation patient-médecin. Est ainsi rendu manifeste le rôle des « garçons », des « élèves », des giovani ou des pupils. Ces termes, couramment utilisés dans les langues française, italienne et anglaise, ne référent pas à un emploi statutaire comme l'apprentissage, mais désignent à la fois la jeunesse et la situation de formation, ou encore la subordination. D'un point de vue quantitatif, les études récentes soulignent l'importance de la main-d'œuvre chez les barbierschirurgiens au XVII ${ }^{\mathrm{e}}$ siècle $^{18}$. Sandra Cavallo a ainsi souligné la part importante des non-licenciés dans la province $(43 \%)$ et à Turin même $(20 \%)^{19}$. Au XVIII ${ }^{\mathrm{e}}$ siècle, l'emploi chirurgical et médical explose dans les hôpitaux, à Paris comme à Londres, mais ne disparaît pas chez les chirurgiens parti-

(17) Sur la mobilité des élèves en chirurgie au XVIII ${ }^{\mathrm{e}}$ siècle, Toby GELFAND, « Deux cultures, une profession : les chirurgiens français au XVIII ${ }^{\mathrm{e}}$ siècle », Revue d'histoire moderne et contemporaine, 27, 1980, p. 468-484; Willem Frijhoff, « L'École de Chirurgie de Paris et les Pays-Bas. Analyse d'un recrutement (1742-1791) », Lias, 17, 1990, p. 185-239; Idem, « Le recrutement étranger de l'Académie Royale de Chirurgie de Paris (1752-1791) : la place des Allemands », dans : Michel Parisse, Les échanges universitaires franco-allemands du Moyen-Âge au XX siècle, Paris : Recherches en civilisations, 1991, p. 73-105; Philip RIEDER, « Médecins et patients à Genève : offres et consommations thérapeutiques à l'époque moderne », Revue d'histoire moderne et contemporaine 52, 2005, p. 61.

(18) Mary K. Gayne, The Wigmakers, the Public, and the State. Cultural and Material Production of Eighteenth-Century French Hairstyles, Phd dissertation, Cornell University, 2006, en particulier chap. 1; Sandra CAvallo, Artisans of the Body in Early Modern Italy : Identities, Families and Masculinities, Manchester : Manchester University Press, 2007, chap. 6, « Age, working relationship and the marketplace », p. 136 et suiv.

(19) Sandra Cavallo, op. cit., p. 225. 
culiers $^{20}$. Les «garçons » tirent leur subsistance de leur salaire en nature ou en argent d'un maître, d'un régiment ou d'un hôpital, sans nécessairement poursuivre leur carrière dans le service médical.

Ainsi la liste de 1807 met-elle à plat, dans les statuts sinon dans l'appréciation des collègues et des patients, une organisation du travail hiérarchisée. Elle la remplace par des nouvelles classes professionnelles : docteurs en médecine et en chirurgie, officiers de santé et sages-femmes.

\section{La nouvelle population médicale}

Le document fait état d'une nouvelle population, que j'ai d'emblée qualifiée de " professionnels de santé » qui proposent des services médicaux. Les pharmaciens en sont exclus : le Collège de pharmacie représente le groupe professionnel le plus stable et le plus autonome tout au long des années révolutionnaires. Comme l'attestent les annuaires, il y a une très forte continuité entre l'ancien et le nouveau régime, l'accès à la députation étant interprété comme un degré supplémentaire dans l'échelle des honneurs ${ }^{21}$.

Pour les praticiens de la médecine et de la chirurgie, l'an XI représente ainsi une ligne de partage entre un ancien et un nouveau régime des professions de la santé. « À partir du $1^{\text {er }}$ vendémiaire an XII, nul ne pourra embrasser la profession de médecin, de chirurgien ou d'officier de santé, sans être examiné et reçu comme il sera prescrit par la présente loi $»^{22}$. La loi du 19 ventôse an XI établit une hiérarchie stricte entre des docteurs en médecine et en chirurgie, d'une part, les sages-femmes d'autre part et, enfin, les officiers de santé, soit trois « ordres ${ }^{23}$. Selon la loi, toux ceux qui obtiendront, à partir du commencement de l'an XII, le droit d'exercer l'art de guérir, porteront le «titre de docteurs en médecine ou en chirur-

(20) Susan C. Lawrence, Charitable Knowledge. Hospital Pupils and Practitioners in Eighteenth-Century London, Cambridge \& New York, Cambridge University Press, 1995, p. 111 et suiv. Sur l'évolution du salariat des chirurgiens, voir Christelle RABIER, Les chirurgiens de Paris et de Londres..., op. cit., chap. 4, « La structure de l'emploi chirurgical », p. 125 et suiv. J'y développe, suivant en cela le modèle proposé par Patrick WaLLis, « Apprenticeship and Training in Pre-Modern England », The Journal of Economic History 68 :3, 2008, p. 832-861, que l'éducation hospitalière a constitué une véritable chance pour les jeunes praticiens, qui ont en conséquence délaissé les boutiques de chirurgiens pour leur prime éducation.

(21) Bibliothèque interuniversitaire de Pharmacie, P. 40117 (1803), Annuaire ou précis des travaux du Collège de Pharmacie de Paris pour l'an XI de la République, Paris, Quillau, an XI.

(22) Loi du 19 ventôse an XI (10 mars 1803), article $1^{\text {er }}$, cité par Jacques LÉONARD, La France médicale..., op. cit., p. 70.

(23) Je reprends ici le terme de Jacques LÉONARD, La France médicale..., op. cit., p. 71, qui n'en distingue que deux. 
gie, lorsqu'ils auront été examinés et reçus dans l'une des six écoles spéciales de médecine, ou celui d'officiers de santé, quand ils seront reçus par les jurys » spécifiques ${ }^{24}$.

Les docteurs forment donc une toute nouvelle catégorie de professionnels. La formation longue - au moins quatre ans - et les examens coûteux - 1000 francs - doivent se dérouler dans une des six Écoles spéciales de médecine - au premier chef, Montpellier, Paris, Strasbourg, fondées en 1795. «Parmi eux, sont choisis les médecins nommés par l'administration dans les hôpitaux et les prisons, désignés pour le jury médical du département, ou retenus par la justice en tant qu'experts » : ils devront servir prioritairement les administrations hospitalière, préfectorale et judiciaire ${ }^{25}$. Ces hommes disposent du droit de s'établir dans toutes les communes de la République, d'enseigner librement et de traiter leurs patients à leur guise ${ }^{26}$. Selon la liste de 1807, il est assez remarquable que le titre de « docteur » continue de désigner pour l'essentiel des praticiens de médecine : ceux-ci forment $93 \%$ des 204 nouveaux « docteurs » sortis des écoles entre l'an XI et l'an XIII.

De ces transformations, Jacques Léonard a précisé le «contexte scientifique », par quoi il entend les attentes professionnelles exprimées dans les cahiers de doléances, qui exigent la réunion de la médecine et de la chirurgie; les transformations de la médecine, sous les auspices de la clinique, de l'anatomie pathologique, de l'auscultation et du vitalisme ${ }^{27}$. Roselyne Rey a cherché à comprendre quelles furent, en pratique, les innovations en matière d'éducation médicale pendant la décennie révolutionnaire $^{28}$. Dans son étude des thèses de doctorat soutenues entre la fin de l'an VII et le début de l'an XII, l'historienne a souligné combien les études médicales étaient tributaires de la formation des chirurgiens d'Ancien Régime. S'il y a mutation professionnelle, c'est plutôt dans la

(24) Loi du 19 ventôse an XI, article 2, cité par Ibid., p. 72.

(25) Jacques LÉONARD, La médecine entre les savoirs et les pouvoirs : histoire intellectuelle et politique de la médecine française au XIX siècle, Paris, Aubier Montaigne, 1981, p. 49.

(26) Jacques LÉONARD, La France médicale..., op. cit., p. 71.

(27) Jacques LÉONARD, La médecine entre les savoirs et les pouvoirs, op. cit., p. 21-42. Létude des cahiers subsistant invite à nuancer sérieusement cette interprétation. Cf. Béatrice Fry Hyslop, Répertoire critique des cahiers de doléances pour les États généraux de 1789, Paris : Ernest Leroux, 1933 ; Idem, Supplément au Répertoire critique des cahiers de doléances pour les États généraux de 1789, Paris, Presses Universitaires de France, 1952, cahiers publiés en partie par Jean-Pierre GOUBERT, 1789 : le corps médical et le changement. Les cahiers de doléances des médecins chirurgiens et apothicaires, Toulouse, Privat, 1984.

(28) Roselyne REY, « L'École de Santé de Paris sous la Révolution. Transformations et innovations », Histoire de l'éducation, 57, 1993, p. 23-57. 
transformation de l'éducation des docteurs en médecine selon un modèle professionnel chirurgical. Sur les 202 thésards qui inscrivent un titre, 140 se disent chirurgiens ou anciens élèves de l'École pratique de dissection; la thèse est conçue comme un écrit venant sanctionner de longues années de pratique, à l'instar de l'acte public de réception au Collège de chirurgie; une partie des élèves sont des auditeurs libres, renouant ainsi avec la fréquentation importante des cours des Écoles de chirurgie; la formation pratique s'effectue dans les trois hospices de l'Humanité - ci-devant Hôtel-Dieu - pour la clinique interne, de l'Unité - ci-devant Charité - pour la clinique externe et de l'École - ci-devant hospice de l'École de chirurgie - pour la clinique de perfectionnement ou des cas rares; les étudiants, dans un tiers des thèses, recourent à l'autopsie pour valider ou invalider des hypothèses sur la nature de la maladie. Toutefois, c'est le titre de docteur en médecine qui est octroyé à la plupart d'entre eux.

Les officiers de santé, quant à eux, représentent une innovation dans la terminologie sinon dans le mode de qualification. Maurice Crosland, dans une étude minutieuse, a mis en évidence comment s'était forgé le nouveau vocable pour désigner ces praticiens de l'art de guérir ${ }^{29}$. L'expression fait référence à la fois aux « officiers » de la monarchie, tenant à la fois des offices civils et militaires, préférée aux «ministres » du service divin. Outre les hommes qui ont exercé l'art de guérir avant 1789, anciens médecins ou chirurgiens, le statut d'officier de santé englobe les jeunes praticiens formés selon des règles spécifiques ${ }^{30}$.

«Les jeunes gens qui se destineront à devenir officiers de santé ne seront pas obligés d'étudier dans les écoles de médecine; ils pourront être reçus officiers de santé, après avoir été attachés, pendant six années, comme élèves, à des docteurs, ou après avoir suivi, pendant cinq années consécutives, la pratique des hôpitaux civils ou militaires. Une étude de trois années consécutives dans les écoles de médecine, leur tiendra lieu de résidence de six années chez les docteurs ou de cinq années dans les hospices $\|^{31}$.

(29) Maurice Crosland, «The Officiers de Santé of the French Revolution : A Case Study in the Changing Language of Medicine », Medical History, 48, 2004, p. 229-244.

(30) Je n'étudierai pas le devenir des sages-femmes ici. Voir Nathalie SAGe-Pranchère, Les sages-femmes en France au XIX siècle, Thèse de doctorat, Université de Paris-IV (en cours) et la publication de sa thèse de l'École des chartes : Nathalie SAGE-Pranchère, Mettre au monde : sagesfemmes et accouchées en Corrèze au XIX siècle, Tulle, Archives départementales de Corrèze, 2007.

(31) Loi du 19 ventôse an XI, article 15, cité par Ibid., p. 71. 
Leur qualification professionnelle héritée de formes anciennes de l'éducation chirurgicale. Elle est fort similaire à celle exigée pour les élèves en chirurgie à partir de 1760 , soit six années d'exercice chez un maître, ou trois dans un hôpital parisien. Si la durée de service sous un maître - les nouveaux «docteurs » - reste identique, le service auprès des hôpitaux civils ou militaires est quasiment doublé, passant de trois à cinq ans. Il s'agit certainement là d'une façon d'alimenter en employés à bas coût des institutions hospitalières qui ne cessent de se développer. Ces mesures renforcent également le rôle des écoles de santé nouvellement mises en place. La réforme dans ce cas est tout autant politique que professionnelle, car il s'agit d'unifier la formation des hommes et des femmes chargés de la santé, mais également de les mettre au service d'une administration hospitalière de la santé toujours en construction. En ce cas pourtant, la rupture date du mitan du XVIII ${ }^{\mathrm{e}}$ siècle, quand les réglementations professionnelles ont élargi les modalités d'accès à la maîtrise, en substituant les années de service hospitalier parisien et de campagnes militaires, aux années d'apprentissage sous un maître.

Le Consulat organise également de nouvelles hiérarchies. Tous ces officiers de santé se trouvent subordonnés aux nouveaux docteurs en médecine et en chirurgie.

« Les officiers de santé ne pourront s'établir que dans le département où ils auront été examinés par le jury, après s'être fait enregistrer comme il vient d'être prescrit. Ils ne pourront pratiquer les grandes opérations chirurgicales que sous la surveillance et l'inspection d'un docteur, dans les lieux où celui-ci sera établi. Dans le cas d'accidents graves arrivés à la suite d'une opération exécutée hors de la surveillance et de l'inspection prescrites ci-dessus, il y aura recours à indemnité contre l'officier de santé qui s'en sera rendu coupable $»^{32}$.

Comme le fait remarquer Jacques Léonard, les officiers de santé se trouvent contraints par une « double entrave, géographique et professionnelle » : ils sont obligés de s'établir dans les arrondissements où ils ont été reçus; « ils ne pourront pratiquer les grandes opérations chirurgicales que sous la surveillance et l'inspection d'un docteur, dans les lieux où celui-ci sera établi $»^{33}$. Le législateur spécifie les modalités d'application

(32) Loi du 19 ventôse an XI, article 29, cité par Jacques LÉONARD, La France médicale..., op. cit., p. 72.

(33) Loi du 19 ventôse an XI, art. 29, cité par Jacques LÉOnARD, Les Médecins de l'Ouest au XIX siècle, thèse de doctorat, Université Paris IV, 1976 (impr. 1978), vol. 1, p. 278. Voir également 
de cette règle : «Dans le cas d'accidents graves arrivés à la suite d'une opération exécutée hors de la surveillance et de l'inspection prescrites ci-dessus, il y aura recours à indemnité contre l'officier de santé s'en sera rendu coupable $»^{34}$.

Le Consulat pose ainsi les bases d'une «biopolitique», terme que Jacques Léonard reprend à Michel Foucault pour désigner une politique étatique de santé. Elle combine ce que Léonard appelle la «professionnalisation de la médecine », un réseau d'institutions d'enseignement médical théorique et pratique, la participation des médecins aux décisions de justice - par la médecine légale - et la mise en place d'une administration de santé publique et d'hygiène, à destination en particulier des femmes et des enfants ${ }^{35}$. Au terme d'une histoire révolutionnaire heurtée, la loi du 19 ventôse an XI vient sanctionner des choix ou amender les conséquences de décisions jugées hasardeuses. Elle crée apparemment de toutes pièces un nouveau régime médical qui a façonné tout le $\mathrm{XIX}^{\mathrm{e}}$ siècle français. Surtout, poursuit l'historien Jacques Léonard, la loi vient mettre fin à la gabegie des années révolutionnaires, au cours desquelles «les intervalles entre les campagnes de guerre ou de mer jettent dans l'exercice de la médecine des officiers de santé sans diplôme qui ont intérêt à voir durer ce désordre institutionnel habillé de principes républicains, et qui sont légion à redouter le passage devant un jury de professionnels $»^{36}$. C'est cette dernière thèse que je souhaiterais examiner maintenant.

\section{La classe 1792-an XI : des praticiens ignorants?}

À coté des docteurs en chirurgie et des officiers de santé reçus suivant les nouvelles formes, les «officiers de santé pourvus de certificats devant leur tenir lieu de diplôme, conformément à l'article 23 de la loi du 19 ventôse an XI » désignent les hommes qui « se sont établis » après la suppression des corporations chirurgicales. Or ces hommes, pour l'essentiel non diplômés faute d'institutions professionnelles à même de le faire, n'ont pour « qualification » que la certification de trois notables de l'arrondissement selon laquelle ces hommes auraient exercé au moins

Ibid., p. 78 : l'historien définit les grandes opérations chirurgicales par la définition qui en est donnée par les docteurs Olliver d'Angers, Velpeau et Adelon, dans « Consultations médico-légales » du 12 août 1840, citée dans les Annales d'hygiène publique, 1841, t. XXV, p. 207.

(34) Loi du 19 ventôse an XI, art. 29, cité par Jacques LÉOnARD, Les Médecins de l'Ouest..., op. cit., vol. 1, p. 281.

(35) Jacques LÉONARD, La médecine entre les savoirs et les pouvoirs, op. cit., p. 46-64.

(36) Ibid., p. 15. 
trois ans dans l'arrondissement. Comment comprendre une telle disposition, contrevenant à toute idée reçue sur la formation médicale?

Selon Jacques Léonard, qui a étudié les textes et les débats à l'origine de la loi du 19 ventôse, « entre le 4 août 1789 et le 25 février 1795, se succèdent des mesures qui détruisent l'ancien régime médical et les trois critères de différenciation, par la nature des études - médicales, chirurgicales, pharmaceutiques -, par le niveau du diplôme obtenu et par le lieu où le praticien est autorisé à exercer $»^{37}$. L'historien en a tracé les grandes lignes : la liberté d'exercer contre le paiement d'un droit de patente, qui ne concerne que les médecins et les chirurgiens à l'exclusion des pharmaciens - loi immédiatement appliquée par les autorités municipales à la recherche de revenus ${ }^{38}-;$ l'abrogation des institutions savantes où s'enseignaient médecine et chirurgie ${ }^{39}$. Si la loi du 8 mars 1793 met à la charge de la nation le paiement des professeurs et l'entretien des bâtiments, plusieurs lois (15 septembre 1793, puis 7 ventôse an III) suppriment « tous les anciens établissements consacrés à l'instruction publique sous le nom de collèges, salariés par la Nation, [...] dans toute l'étendue de la République $\gg^{40}$. L'application de la loi a varié selon les situations locales. Pour ne prendre que l'exemple de la chirurgie, dans l'Ouest, son enseignement a mieux résisté que la médecine. Le Collège de Rennes n'est pas supprimé avant germinal an III, enregistre les étudiants, perçoit des droits d'inscription, distribue les fonctions de chirurgiens de l'hôpital et des

(37) Voir Jacques LÉOnARD, Les Médecins de l'Ouest..., op. cit., vol. 1, chap. II, « Les médecins et la Révolution », p. 196-252 et chap. III, « La loi de ventôse an XI et le nouvel ordre social », p. 253-302 et Jacques LÉONARD, La médecine entre les savoirs et les pouvoirs, op. cit., p. 11-66, citation p. 14.

(38) Loi du 2 mars 1791 : voir Jacques LÉOnARD, Les Médecins de l'Ouest..., op. cit., vol. 1, p. 217. Sur le droit de patente imposé aux professions de santé comme aux autres métiers, les débats devant l'Assemblée indiquent l'inclusion des professions de santé dans le cadre de la loi sur les patentes comme un mode de participation à l'effort national, à l'initiative des médecins parlementaires eux-mêmes. Elle fut aussitôt discutée par les médecins parisiens. Sur l'abolition des corporations, voir Liana VARDI, «The Abolition of the Guilds during the French Revolution », French Historical Studies, XV, 1988, p. 704-717.

(39) Lois du 18 août 1792, du 14 mars et du 15 septembre 1793 : voir Jacques LÉONARD, Les Médecins de l'Ouest..., op. cit., vol. 1, p. 217-219. Bien que les textes concernent au premier chef les congrégations ecclésiastiques, ils visent également l'enseignement des collèges et confréries et le service hospitalier, dorénavant dispensé à titre individuel et non plus collectif, et pris en charge par la Nation. Léonard précise que la loi du 3 août 1793 a contribué à affaiblir les établissements, avant le texte du 3 ventôse an III, qui supprime « tous les établissements consacrés à l'instruction publique sous le nom de collèges, salariés par la Nation ». L'historien a établi que les professeurs de l'Ouest ont continué leurs cours, interrompus seulement par la guerre civile.

(40) Ibid., vol. 1, p. 219. La faculté de Caen continue d'abriter des cours et d'octroyer des diplômes. 
prisons, de prévôt, de trésorier, d'inspecteurs, de « chirurgien consultant des pauvres ». «Le décret du $1^{\text {er }}$ août 1793 leur confère même une tâche nouvelle : l'examen des chirurgiens pour les armées. Le Collège se réunit donc pour cela au moins cinq fois $\rangle^{41}$. Le 6 germinal an III, le collège prononce sa dissolution « avec une vraie et vive douleur », indique le trésorier Maugé : il se soumet au décret «par patriotisme $»^{42}$. Quant à la disparition de la corporation parisienne, elle fut plus précoce. Il semble que dès la fin de l'année 1790, la corporation se trouve dans une impasse et réclame des subsides de l'Académie : «M. Disdier a dit que depuis un an les réceptions de maîtres experts et sages-femmes etoient presque nulles : d'où il résulte que les fonds de la bourse commune se trouvent presqu'entièrement épuisés, et que le Collège est dans le plus grand embarras pour l'acquit de ses rentes $\gg{ }^{43}$. Le bureau de l'Académie refuse, prétextant que la fondation du Premier chirurgien La Peyronie n'était pas destinée aux maîtres. À la mort du secrétaire de l'Académie royale de chirurgie, les prévôts du Collège se battent pour conserver leur bibliothèque que Louis avait fondue pendant de longues années avec la sienne ${ }^{44}$. La mort des institutions savantes, le 8 août 1793, sonne le glas de l'Académie royale de chirurgie, alors que le Collège était déjà moribond.

Dans l'architecture de la loi de ventôse an XI, les hommes formés à la médecine ou à la chirurgie après 1792 forment ainsi une classe problématique. L'apparition d'une nouvelle catégorie statutaire, véritable nuisance selon l'historien Léonard, trouverait sa raison dans le manque d'hommes. De fait, la question démographique, aux yeux des législateurs, est de taille. Depuis le début de la guerre, comme le rapporte Fourcroy devant la Convention nationale, la profession médicale déplore de lourdes pertes, qui ne sont pas compensées par la formation de nouveaux officiers de santé : « La Convention apprendra avec sensibilité que plus de six cents officiers de santé ont péri depuis dix-huit mois $»^{45}$. L'enjeu est donc

(41) Ibid., vol. 1, p. 220

(42) BM Rennes, 4082, «Registre de délibérations du collège des chirurgiens de Rennes, 1791-1793 », cité par Ibid., vol. 1, p. 221.

(43) Académie nationale de médecine (désormais Acad Med), MS 17, « $3^{\mathrm{e}}$ registre du fonds d'administration de l'Académie royale et de l'hospice de chirurgie », fol. 269, 30 décembre 1790 .

(44) Acad Med, fonds Académie royale de chirurgie, cart. 60, doss. 2, fol. 194, Sue, Chopart et Peyrihle, « Memoire à consulter pour le Collège et l'Académie de chirurgie sur les dispositions qui les concernent dans les testamens de M. Louis », 10 juillet 1792.

(45) BNF, LE38-1060, Antoine FouRCROY, Rapport et projet de décret sur l'établissement d'une École centrale de santé à Paris, fait à la Convention nationale, au nom des Comités de salut public et d'instruction publique, le 7 frimaire an III, Paris, Imprimerie nationale, an III [1794], p. 2. Roselyne ReY, op. cit., p. 32, indique les pertes signalées dans l'Encyclopédie méthodique (art. 
politique : il s'agit de s'attacher des hommes qui ont montré très tôt leur attachement à la patrie en s'enrôlant massivement. La nouvelle loi, selon Léonard - et je le suis sur ce point - aurait pour finalité de limiter l'exclusion de praticiens déjà formés en exigeant d'eux un examen que le coût pourrait rebuter mais également de reconnaître le prix du sang versé.

Faut-il y lire la réalité d'une formation insuffisante? Ce serait méconnaître les modalités de la formation professionnelle du XVIII ${ }^{\mathrm{e}}$ siècle et de ses évolutions : l'éducation professionnelle ne peut se résumer à la dispense de cours théoriques, quand bien même ceux-ci n'auraient pas été dispensés de façon régulière après la suppression des corporations médicales. Léonard, lui-même, établit que, dans l'Ouest, l'enseignement de la médecine et de la chirurgie, ainsi que la délivrance de diplômes et de certificats de validation de cours, se poursuivent entre 1789 et 1803 , malgré quelques interruptions. Une série d'études ont depuis établi les fortes continuités entre l'Ancien Régime médical et le début du XIX ${ }^{\mathrm{e}}$ siècle ${ }^{46}$. Laurence Brockliss a le plus fermement contesté l'idée d'une révolution médicale par le haut : selon lui, les cinq examens oraux du doctorat établis en l'an XI sanctionnent, mais n'évaluent pas véritablement la formation antérieure; l'enseignement clinique institué pendant la Révolution ne serait pas mis en œuvre, sinon dans les cours privés, comme ceux d'un Poumiès de la Siboutie ou d'un Capuron; l'École pratique de dissection, ouverte de nouveau en 1797 après trois ans de fermeture, a du mal à remplir ses cent vingt places annuelles, signe selon l'historien britannique que peu d'étudiants voulaient s'astreindre à la rigueur de cette formation approfondie $^{47}$. Toutefois, Brockliss nuance l'idée d'absence d'innovation : « Contrairement à la période antérieure à 1789, la possibilité d'acquérir une expérience pratique au sein d'un service hospitalier n'est plus uniquement déterminée par le réseau des connaissances de l'étudiant », alors que le système d'externat et d'internat est établi en1802. Cela est vrai pour les

« Médecine », t. 9,1816, p. 270-292) à partir desquels il est possible de calculer un taux de mortalité qui s'avère colossal pendant les guerres du Consulat et de l'Empire : $26 \%$ pour l'an VIII, $29 \%$ pour l'an IX et $40 \%$ pour l'an XII.

(46) Pierre Huard et Marie-José Imbault-Huart, « Concepts et réalités de l'éducation et de la profession médico-chirurgicale pendant la Révolution », Journal des savants, 1973, p. 126-150; Lawrence W. BRockLISs, « L'enseignement médical et la Révolution : essai de réévaluation », Histoire de l'éducation, 42, mai 1989, 79-110; Roselyne REY, op. cit., p. 23-57 : 1'historienne entre davantage dans l'étude de la nouveauté des études médicales révolutionnaires.

(47) Laurence W. Brockliss, op. cit., p. 101-104. Sur l'histoire des bâtiments de l'École, voir Marie-José Imbault-Huart, L'École Pratique de Dissection de Paris de 1750 à 1822 ou l'influence du concept de médecine pratique et de médecine d'observation dans l'enseignement médicochirurgical au XVIII et au début du XIX siècle, Thèse de doctorat, Université de Paris-I, 1973. 
étudiants en médecine : quant aux élèves en chirurgie, Toby Gelfand avait déjà démontré que des places étaient offertes par concours à partir du milieu du XVIII ${ }^{\mathrm{e}}$ siècle dans les hôpitaux parisiens ${ }^{48}$. C'est donc là, et dans l'explosion de la formation pratique hospitalière à partir des années 1740 , que se joue une des clefs de la rupture révolutionnaire, définitivement établie par la loi de ventôse an $\mathrm{XI}^{49}$.

L'autre disposition de la loi constituerait un indice flagrant de l'incompétence de la classe 1792-an XI : la qualification des officiers de santé s'est trouvée seulement vérifiée par le maire de leur arrondissement et deux notables désignés par le sous-préfet. Bien qu'il ne s'agisse pas d'une certification par les pairs, elle ne doit pas être sous-estimée. Elle repose sur l'idée que les praticiens en exercice avaient déjà reçu une certaine éducation, et que la sanction par voie d'examen aurait été contre-productive. Chaptal ne le dit pas autrement : dans une circulaire du 13 fructidor an XI, il indique que « la loi certainement n'a pas entendu favoriser les hommes qui n'auraient acquis aucune instruction »; elle a seulement « des égards pour la position où un grand nombre d'individus se trouvent par l'effet de la révolution»; «il ne convenait pas d'exiger d'eux la preuve de leurs connaissances par des examens auxquels ils seraient appelés $\|^{50}$.

Allons plus loin : la certification par les notables pouvait constituer un indice suffisant de qualification professionnelle. C'est ce qui ressort de l'étude de Sandra Cavallo sur Turin. S'y déroule, un siècle plus tôt, une enquête sur la légalité de pratique des chirurgiens à l'initiative du fisc. Les archives de la Consegna de 1695 permettent de comprendre pourquoi la reconnaissance des populations locales constituait un critère suffisant de qualification $^{51}$. Selon les justifications fournies dans les dépositions, certains praticiens « illégaux » seulement envisageaient l'acquisition d'une licence comme une étape normale de carrière, retardée par la guerre ou la pauvreté. La subordination effective à un chirurgien ou un médecin dûment licencié rendait d'ailleurs caduque la nécessité d'en posséder. Toutefois, de nombreux chirurgiens, à l'instar de Giò Matteo Ferraris, qui déclare « qu'il a pratiqué pendant trente ans sans avoir de licence, qu'il a toujours exercé

(48) Toby Gelfand, Professionalizing Modern Medicine: Paris Surgeons and Medical Science and Institutions in the Eighteenth Century, Wesport, Conn., Greenwood Press, 1980, p. $108-113$.

(49) Voir la note 20.

(50) Cité par Jacques LéonARd, Les Médecins de l'Ouest..., op. cit., vol. 1, p. 273.

(51) Sandra Cavallo, op. cit., chap. 10, « The good surgeon », p. 225 et suiv. 
son métier sans aucune contestation », l'absence de licence ne signifie pas incompétence ou défaut de crédibilité. Le praticien acquiert sa respectabilité par la longévité de résidence et la confiance que lui accordent ses patients. On peut ainsi argumenter que la reconnaissance de notables, en l'absence d'autres formes de sanction d'études, pouvait constituer un critère suffisant pour les autorités. La certification de trois années de pratique par des notables, dont le maire, qui valide la longévité dans l'arrondissement et la confiance du public a pu suffire pour déterminer quels hommes constituaient des «professionnels de santé ».

La liste établie par la Préfecture de Paris en 1807 permet de comprendre les évolutions qui se sont fait jour au sein des praticiens de médecine et de chirurgie au tournant $\mathrm{du} \mathrm{XIX}^{\mathrm{e}}$ siècle. Contrairement aux perspectives adoptées par les historiens de la médecine, la discontinuité révolutionnaire ne porte peut-être pas tant sur les progrès du savoir, que sur les modalités d'agrégation professionnelle.

À partir de 1807, l'administration ne reconnaît plus que deux catégories de professionnels - les docteurs et les officiers de santé, si l'on passe sous silence les sages-femmes et les pharmaciens. Autorisant l'établissement d'un plus grand nombre de praticiens, ces dispositions mettent en contrepartie officiellement fin à un important salariat médical, longtemps chasse gardée des docteurs en médecine et des maîtres en chirurgie. La véritable rupture - la disparition du « garçon » - s'est produite au mitan $\mathrm{du} \mathrm{XVIII}^{\mathrm{e}}$ siècle, quand la monarchie a pris des dispositions pour réserver l'emploi médical à bas coût aux hôpitaux parisiens et aux armées. En encourageant les jeunes praticiens à donner les premières années de carrière au service de l'institution hospitalière et militaire, l'administration impériale moissonne les résultats d'une politique dont le succès ne s'est plus démenti après 1740 . On peut, à l'instar d'Erwin H. Ackerknecht, parler de « médecine hospitalière » si l'on comprend qu'elle n'est pas apparue avec la Révolution, mais s'est confirmée après 1800, stabilisant une récente forme d'accession au métier de médecin ou de chirurgien ${ }^{52}$. Outre l'assèchement du travail salarié au profit des pauvres et des soldats, c'est également le statut des veuves qui se trouve vidé de son contenu, ce qui

(52) Erwin H. Ackerknecht, Medicine at the Paris Hospital, 1794-1848, Baltimore, The Johns Hopkins University Press, 1967. 
a eu sans nul doute des conséquences sur la cellule économique familiale mise en évidence pour l'Ancien Régime : la fin des jurandes met un terme aux formes d'assistance familiale mises en place sous l'Ancien Régime. Enfin, par les dispositions de la loi du 19 ventôse an XI, l'État se dote d'une administration de la santé qui peut puiser dans le vivier des docteurs et se substitue aux anciennes corporations dans la lutte contre le travail illégal.

Pourtant, bien qu'on ne puisse nier qu'avec la loi de ventôse an XI, se mette en place une nouvelle organisation professionnelle de l'art de guérir, il convient de souligner les fortes continuités avec la profession chirurgicale d'Ancien Régime. Tout d'abord, la nouvelle profession médicale intègre tous les praticiens qui ont commencé leurs études avant la suppression des corporations. Du point de vue de l'éducation, ensuite, la loi de ventôse reprend sans presque rien changer les pratiques des Lumières et $\mathrm{y}$ ajoute un doctorat pour ceux auxquels le gouvernement veut confier les tâches de l'administration de la santé, de l'expertise médico-légale aux postes d'officiers publics. Au vu des premiers diplômés, il apparaît que le doctorat reste l'apanage des praticiens de médecine, associant durablement le nom de médecin à celui de « docteur ». Pour autant, les évolutions des pratiques professionnelles se fondent sur la foi dans le progrès de la connaissance médicale et organisent des nouveaux modes de communication, en partie hérités de la République des lettres, mais tout de même résolument nouveaux. Les échanges de correspondance, les périodiques médicaux en constituent les principales caractéristiques ${ }^{53}$.

On peut être étonné de la faible résistance des professions médicales à ces transformations statutaires : y aurait-il eu adhésion aux réformes que certains des membres de la profession - Fourcroy au premier chef ont dessinées? Rien n'est moins sûr. Il est certain que les législateurs se sont attachés à soutenir les praticiens formés pendant les années de guerre en facilitant leur installation. Ils ont aussi sans doute adhéré aux modalités de légitimation reconnues aux praticiens médicaux par les sociétés urbaines. Outre la rémanence de l'organisation professionnelle dont je viens de parler, on peut avancer d'autres hypothèses pour expliquer cette inertie : d'abord, la suppression du droit de patente, globalement accepté

(53) William F. Bynum, Stephen Lock et Roy Porter (éd.), Medical Journals and Medical Knowledge : Historical Essays, London \& New York, Routledge, 1992 et Christelle RABIER, Les chirurgiens de Paris et de Londres..., op. cit., p. 396 et suiv. et « La disparition du barbier-chirurgien : analyse d'une mutation professionnelle au $18^{\mathrm{e}}$ siècle », Annales Histoire, Sciences sociales, 2010 (à paraître). 
par les praticiens tant qu'il s'agissait de s'associer à l'effort national, mais rapidement mis en cause; peut-être aussi le soutien des officiers de santé qui n'ont pas passé d'examen; enfin, la transformation des modes de sociabilités professionnelles.

Christelle RABIER

Institut d'histoire moderne et contemporaine 45 , rue d'Ulm 75005 Paris christelle.rabier@gmail.com 